\title{
Stationary Distribution and Extinction of a Stochastic SIQR Model with Saturated Incidence Rate
}

\author{
Qiuhua Zhang and Kai Zhou $\mathbb{D}$ \\ School of Mathematics and Computers, Chizhou University, Chizhou 247000, China \\ Correspondence should be addressed to Kai Zhou; zk1984@163.com
}

Received 3 January 2019; Revised 26 March 2019; Accepted 10 June 2019; Published 24 June 2019

Academic Editor: Leonid Shaikhet

Copyright (c) 2019 Qiuhua Zhang and Kai Zhou. This is an open access article distributed under the Creative Commons Attribution License, which permits unrestricted use, distribution, and reproduction in any medium, provided the original work is properly cited.

In this paper, we consider a stochastic SIQR epidemic model with saturated incidence rate. By constructing a proper Lyapunov function, we obtain the existence and uniqueness of positive solution for this SIQR model. Furthermore, we study the dynamical properties of this stochastic SIQR model; that is, (i) we establish the sufficient condition for the existence of ergodic stationary distribution of the model; (ii) we obtain the extinction of the disease under some conditions. At last, numerical simulations are introduced to illustrate our theoretical results.

\section{Introduction}

Due to the importance of disease transmission and control, epidemic models are attracting more and more attention from scholars. Since the pioneer work of Kermack and McKendrick (see [1]), where they described a directly transmitted viral or bacterial agent in a closed population and divided the total population into three subparts as susceptibles $(S)$, infectives $(I)$, and recovers $(R)$, mathematical models have proven their adequacy to describe and analyze the propagation and control of infectious diseases. From then on, various epidemic models have been developed in recent decades, such as SIR models, SIS models, and SEIR models; see [2-6].

In modern society, due to the close interpersonal communication, the harm of disease transmission is more and more significant, and various measures have been taken to control the spread of disease. Among them, isolation measure was widely used by public health institutions and governments to control the spread of disease. In reality, it has been proved that the isolation measure is an effective method to suppress disease such as leprosy, plague, cholera, smallpox, tuberculosis, measles, rabies, foot and mouth, and ebola, which has ravaged West Africa in recent years [7]. Therefore, from the point of view of mathematic, the quarantine subpopulation (Q) was introduced into the epidemic models, and SIQ, SIQS, SIQR models have been proposed in the literature [810]. Afterwards Hethcote et al. [11] analyzed six SIQS and
SIQR models with bilinear, standard, or quarantine-adjusted incidence and found that a Hopf bifurcation might occur in the SIQR model with quarantine-adjusted incidence rate. Recently, Nirwani et al. [12] considered a SIQR epidemic model with saturated incidence rate as follows:

$$
\begin{aligned}
& \frac{d S(t)}{d t}=\Lambda-\frac{\beta S(t) I(t)}{1+\alpha I(t)}-\mu S(t), \\
& \frac{d I(t)}{d t}=\frac{\beta S(t) I(t)}{1+\alpha I(t)}-\left(\delta+\gamma+\mu+\mu_{1}\right) I(t), \\
& \frac{d Q(t)}{d t}=\delta I(t)-\left(\kappa+\mu+\mu_{2}\right) Q(t), \\
& \frac{d R(t)}{d t}=\gamma I(t)+\kappa Q(t)-\mu R(t),
\end{aligned}
$$

where they found that the equilibrium points are locally asymptotically stable under certain conditions.

Recently, some researchers have considered the impact of environmental noise on the dynamics of epidemic models [13-16]. In reality, the environmental noise may involve the variations of factors such as climate, habitats, health habits, medical quality, and so on, which may affect the natural birth rate, death rate, and so on [17]. Especially, for human diseases, the nature of epidemic growth and spread is inherently stochastic, as the contacts of person-to-person 
are unpredictable [18]. Therefore, stochastic models may be a more accurate method to describe the spread of infectious diseases in humans (see, e.g., [19-26]). There are various approaches to include random perturbation in epidemic models, and among them, parameter perturbation method is a frequently adopted way. In this paper, we will adopt the method of Cai et al. [27]; that is, we replace $-\mu d t$ in (1) with $-\tilde{\mu} d t=-\mu d t+\sigma_{i} d B_{i}(t)(i=1,2,3,4)$. Therefore, we propose the following stochastic SIQR epidemic model with saturated incidence:

$$
\begin{aligned}
d S(t)= & \left(\Lambda-\frac{\beta S(t) I(t)}{1+\alpha I(t)}-\mu S(t)\right) d t+\sigma_{1} S d B_{1}(t), \\
d I(t)= & \left(\frac{\beta S(t) I(t)}{1+\alpha I(t)}-\left(\delta+\gamma+\mu+\mu_{1}\right) I(t)\right) d t \\
& +\sigma_{2} I d B_{2}(t), \\
d Q(t)= & \left(\delta I(t)-\left(\kappa+\mu+\mu_{2}\right) Q(t)\right) d t+\sigma_{3} Q d B_{3}(t), \\
d R(t)= & (\gamma I(t)+\kappa Q(t)-\mu R(t)) d t+\sigma_{4} R d B_{4}(t),
\end{aligned}
$$

where all parameters are positive. For the biological meaning of each coefficient, we briefly mention below: $\Lambda$ is the recruitment rate of the population, $\beta$ is the average number of adequate contacts of a person per unit time, $\alpha$ measures the appropriate preventions taken by infectious for epidemic control, and $\mu$ is the natural death rate of the population, while $\mu_{1}, \mu_{2}$ are the extra disease-related death rate in compartments $I$ and $Q$, respectively. $\gamma, \kappa$ denote the recover rates from group $I, Q$ to $R$, respectively, and $\delta$ denotes the removal rate from $I$. Additionally, $B_{1}(t), B_{2}(t), B_{3}(t)$, and $B_{4}(t)$ are standard one-dimensional independent Brownian motions, and $\sigma_{i}^{2}>0$ are the intensities of the white noises, $i=1,2,3,4$.

This paper is organized as follows. In Section 2, we give some preliminaries and notations which will be used in the following analysis. In Section 3, we prove that there is a unique global positive solution of system (2). In Section 4, we show the main result that there is an ergodic stationary distribution of system (2) under certain conditions. In Section 5, we establish sufficient conditions for extinction of the disease. Some numerical simulations are given to illustrate the analytical result in Section 6. Finally, we provide a brief summary of the main results and put forward some research prospects.

\section{Preliminaries and Some Notations}

Throughout this paper, let $(\Omega, \mathscr{F}, \mathbb{P})$ be a complete probability space with a filtration $\left\{\mathscr{F}_{t}\right\}_{t \geq 0}$, which satisfies the usual conditions (i.e., it is increasing and right continuous while $\mathscr{F}_{0}$ contains all $\mathbb{P}$-null stes) and $B_{i}(t)(i=1,2,3,4)$ are defined on this complete probability space. Denote $\mathbb{R}_{+}^{d}=\left\{x \in \mathbb{R}^{d}\right.$ : $\left.x_{i}>0,1<i<d\right\}$ and $a \vee b:=\max \{a, b\}, a \wedge b:=\min \{a, b\}$.

For the $d$-dimensional stochastic differential equation

$$
d X(t)=f(X(t), t) d t+g(X(t), t) d B(t)
$$

$$
\text { for } t \geq 0 \text {, }
$$

with initial value $X(0)=X_{0} \in \mathbb{R}^{d}$, while $B(t)$ is a $d$-dimensional standard Brownian motion defined on the complete probability space $\left(\Omega, \mathscr{F},\left\{\mathscr{F}_{t}\right\}_{t \geq 0}, \mathbb{P}\right)$, we define the differential operator $L$ of (3) as follows:

$$
\begin{aligned}
L= & \frac{\partial}{\partial t}+\sum_{i=1}^{d} f_{i}(X, t) \frac{\partial}{\partial X_{i}} \\
& +\frac{1}{2} \sum_{i, j=1}^{d}\left[g^{T}(X, t) g(X, t)\right]_{i j} \frac{\partial^{2}}{\partial X_{i} \partial X_{j}} .
\end{aligned}
$$

For $V(X, t) \in C^{2,1}\left(\mathbb{R}^{d} \times[0, \infty) ; \mathbb{R}_{+}\right)$, which represents the family of nonnegative functions defined on $\mathbb{R}^{d} \times[0, \infty)$ such that they are continuously twice differentiable in $X$ and once in $t$, by acting $L$ on this function $V$, we have

$$
\begin{aligned}
L V(X, t)= & V_{t}(X, t)+V_{X}(X, t) f(X, t) \\
& +\frac{1}{2} \operatorname{trace}\left[g^{T}(X, t) V_{X X}(X, t) g(X, t)\right] .
\end{aligned}
$$

By the Itô's formula, if $X(t) \in \mathbb{R}^{d}$, then

$$
\begin{aligned}
d V(X(t), t)= & L V(X(t), t) d t \\
& +V_{X}(X(t), t) g(X(t), t) d B(t) .
\end{aligned}
$$

At last, we give some theory about the stationary distribution. Firstly, we recall the definitions of homogeneous Markov process and stationary distribution.

Definition 1 (see [28]). The transition probability function $P(s, t, x, A)$ is said to be time-homogeneous (and the corresponding Markov process is called time-homogeneous) if the function $P(s, t, x, A)$ is independent of $s$, where $0 \leq s \leq t, x \in$ $\mathbb{R}^{d}$, and $A \in \mathscr{B}$ and $\mathscr{B}$ denotes the $\sigma$-algebra of Borel sets in $\mathbb{R}^{d}$.

Definition 2 (see [29]). Denote $\mathbb{P}_{\gamma}$ the corresponding probability distribution of an initial distribution $\gamma$, which describes the initial state of (3) at $t=0$. Suppose that the distribution of $X(t)$ with initial distribution $\gamma$ converges in some sense to a distribution $\pi=\pi_{\gamma}$, i.e.,

$$
\lim _{t \rightarrow \infty} \mathbb{P}_{\gamma}\{X(t) \in F\}=\pi(F)
$$

for all measurable $F$, then we say that (3) has a stationary distribution $\pi(\cdot)$.

Let $X(t)$ be a homogeneous Markov process in $E_{d}$ and satisfy the following stochastic differential equation:

$$
d X(t)=b(X) d t+\sum_{r=1}^{k} g_{r}(X) d B_{r}(t)
$$

The diffusion matrix is denoted as $A(x)=\left(a_{i j}(x)\right)$, where $a_{i j}(x)=\sum_{r=1}^{k} g_{r}^{i}(x) g_{r}^{j}(x)$. 
Lemma 3 (see [28]). The Markov process $X(t)$ has a unique ergodic stationary distribution $\pi(\cdot)$ if there exists a bounded domain $D \subset E_{d}$ with regular boundary $\Gamma$ and

$A_{1}$ : in the open domain $D$ and some neighborhood thereof, the smallest eigenvalue of the diffusion matrix $A(x)$ is bounded away from zero;

$A_{2}:$ if $x \in E_{d} \backslash D$, the mean time $\tau$ which a path issuing from $x$ reaches the set $D$ is finite, and $\sup _{x \in K} E_{x} \tau<\infty$ for every compact subset $K \in E_{d}$.

Then

$$
\mathbb{P}_{x}\left\{\lim _{T \rightarrow \infty} \frac{1}{T} \int_{0}^{T} f(X(t)) d t=\int_{E_{d}} f(x) \pi(d x)\right\}=1
$$

for all $x \in E_{d}$, where $f(\cdot)$ is a function integrable with respect to the measure $\pi$.

Remark 4. As pointed in [24], to validate $A_{1}$, we need to prove

$\widetilde{A}_{1}$ : there is a positive number $M$ such that $\sum_{i, j=1}^{d} a_{i j}(x) \xi_{i} \xi_{j} \geq$ $M|\xi|^{2}, x \in D, \xi \in \mathbb{R}^{d}$; and to validate $A_{2}$, we need to prove

$\widetilde{A}_{2}$ : there exists a nonnegative $C^{2}$ - function $V$ such that $L V$ is negative for any $E_{d} \backslash D$. Thus, we will verify the conditions $\widetilde{A}_{1}$ and $\widetilde{A}_{2}$ to obtain the existence of stationary distribution for Markov process $X(t)$.

\section{Existence and Uniqueness of the Positive Solution}

In this section, we will give the existence and uniqueness of positive solutions for system (2), which is the basis of studying the long-time behavior of the model.

Theorem 5. For any initial value $X_{0}=\left(S_{0}, I_{0}, Q_{0}, R_{0}\right) \in \mathbb{R}_{+}^{4}$, there is a unique positive solution $X(t)=(S(t), I(t), Q(t), R(t))$ of system (2) on $t \geq 0$ and the solution will maintain in $\mathbb{R}_{+}^{4}$ with probability one.

Proof. The proof is motivated by the work Mao et al. [30]. Since the coefficients of system (2) satisfy the local Lipschitz condition, then for any initial value $\left(S_{0}, I_{0}, Q_{0}, R_{0}\right) \in \mathbb{R}_{+}^{4}$, there exists a unique local solution $(S(t), I(t), Q(t), R(t))$ on $\left[0, \tau_{e}\right)$, where $\tau_{e}$ is the explosion time [31]. To verify that this solution is global, we only need to prove that $\tau_{e}=\infty$ a.s. For this purpose, let $n_{0}>0$ be large enough such that every component of $X_{0}$ lay within the interval $\left[1 / n_{0}, n_{0}\right]$. For each integer $n>n_{0}$, define the following stopping time:

$$
\begin{aligned}
\tau_{n} & =\inf \left\{t \in\left[0, \tau_{e}\right): \min \{(S(t), I(t), Q(t), R(t))\}\right. \\
& \left.\leq \frac{1}{n} \text { or } \max \{(S(t), I(t), Q(t), R(t))\} \geq n\right\} .
\end{aligned}
$$

For empty set $\Phi$, we define $\inf \Phi=\infty$.

It is obvious that $\tau_{n}$ is increasing with $n \longrightarrow \infty$. Let $\tau_{\infty}=$ $\lim \sup _{n \rightarrow \infty} \tau_{n}$, then $\tau_{\infty} \leq \tau_{e}$ a.s. In the following, we only need to prove that $\tau_{\infty}=\infty$ a.s. If this assertion is not valid, then there is a pair of constants $T>0$ and $\epsilon \in(0,1)$ such that
$\mathbb{P}\left\{\tau_{\infty} \leq T\right\}>\epsilon$. Hence, there exists an integer $n_{1} \geq n_{0}$ such that

$$
\mathbb{P}\left\{\tau_{n} \leq T\right\} \geq \epsilon, \quad n \geq n_{1}
$$

Define a $C^{2}$ - function $V: \mathbb{R}_{+}^{4} \longrightarrow \mathbb{R}_{+}$as follows:

$$
\begin{aligned}
V(S, I, Q, R)= & (S-1-\ln S)+(I-1-\ln I) \\
& +(Q-1-\ln Q)+(R-1-\ln R) .
\end{aligned}
$$

Utilizing the Itô's formula, we get that

$$
\begin{aligned}
d V(S, I, Q, R)= & L V(S, I, Q, R) d t+\sigma_{1}(S-1) d B_{1}(t) \\
& +\sigma_{2}(I-1) d B_{2}(t) \\
& +\sigma_{3}(Q-1) d B_{3}(t) \\
& +\sigma_{4}(R-1) d B_{4}(t),
\end{aligned}
$$

where

$$
\begin{aligned}
& L V=\left(1-\frac{1}{S}\right)\left(\Lambda-\frac{\beta S I}{1+\alpha I}-\mu S\right) \\
& +\left(1-\frac{1}{I}\right)\left(\frac{\beta S I}{1+\alpha I}-\left(\delta+\gamma+\mu+\mu_{1}\right) I\right) \\
& +\left(1-\frac{1}{Q}\right) \times\left(\delta I-\left(\kappa+\mu+\mu_{2}\right) Q\right) \\
& +\left(1-\frac{1}{R}\right)(\gamma I+\kappa Q-\mu R) \\
& +\frac{\sigma_{1}^{2}+\sigma_{2}^{2}+\sigma_{3}^{2}+\sigma_{4}^{2}}{2} \\
& =\Lambda+4 \mu+\delta+\gamma+\kappa+\mu_{1}+\mu_{2} \\
& -\mu(S+I+Q+R)-\mu_{1} I-\mu_{2} Q+\frac{\beta I}{1+\alpha I} \\
& -\frac{\beta S}{1+\alpha I}-\frac{\Lambda}{S}-\frac{\delta I}{Q}-\frac{\gamma I}{R}-\frac{\kappa Q}{R} \\
& +\frac{\sigma_{1}^{2}+\sigma_{2}^{2}+\sigma_{3}^{2}+\sigma_{4}^{2}}{2} \\
& <\Lambda+4 \mu+\left(\delta+\gamma+\kappa+\frac{\beta}{\alpha}+\mu_{1}+\mu_{2}\right) \\
& +\frac{\sigma_{1}^{2}+\sigma_{2}^{2}+\sigma_{3}^{2}+\sigma_{4}^{2}}{2}:=G,
\end{aligned}
$$

where $G$ is a positive constant which is independent of $S, I, Q, R$, and $t$. Therefore,

$$
\begin{aligned}
d V(S, I, Q, R) \leq & G d t+\sigma_{1}(S-1) d B_{1}(t) \\
& +\sigma_{2}(I-1) d B_{2}(t) \\
& +\sigma_{3}(Q-1) d B_{3}(t) \\
& +\sigma_{4}(R-1) d B_{4}(t) .
\end{aligned}
$$

Integrating both sides of (15) from 0 to $\tau_{n} \wedge T$, then 


$$
\begin{aligned}
& V\left(S\left(\tau_{n} \wedge T\right), I\left(\tau_{n} \wedge T\right), Q\left(\tau_{n} \wedge T\right), R\left(\tau_{n} \wedge T\right)\right) \\
& \quad-V\left(S_{0}, I_{0}, Q_{0}, R_{0}\right) \leq G\left(\tau_{n} \wedge T\right) \\
& \quad+\int_{0}^{\tau_{n} \wedge T}\left[\sigma_{1}(S-1) d B_{1}(t)+\sigma_{2}(I-1) d B_{2}(t)\right. \\
& \left.\quad+\sigma_{3}(Q-1) d B_{3}(t)+\sigma_{4}(R-1) d B_{4}(t)\right]
\end{aligned}
$$

Further, taking the expectation for the above inequality and due to the property of Brownian motion, we obtain

$$
\begin{aligned}
& \mathbb{E} V\left(S\left(\tau_{n} \wedge T\right), I\left(\tau_{n} \wedge T\right), Q\left(\tau_{n} \wedge T\right), R\left(\tau_{n} \wedge T\right)\right) \\
& \quad \leq V\left(S_{0}, I_{0}, Q_{0}, R_{0}\right)+G T .
\end{aligned}
$$

Let $\Omega_{n}=\left\{\tau_{n} \leq T\right\}$ for $n \geq n_{1}$. By (11) we have $\mathbb{P}\left(\Omega_{n}\right) \geq \epsilon$. From the definition of the stopping time, we can deduce that, for every $\omega \in \Omega_{n}, S\left(\tau_{n}, \omega\right)$ or $I\left(\tau_{n}, \omega\right)$ or $Q\left(\tau_{n}, \omega\right)$ or $R\left(\tau_{n}, \omega\right)$ equals either $n$ or $1 / n$. Hence, it follows from (17) that

$$
\begin{aligned}
& V\left(S_{0}, I_{0}, Q_{0}, R_{0}\right)+G T \geq \mathbb{E}\left[1 _ { \Omega _ { n } ( \omega ) } V \left(S\left(\tau_{n}, \omega\right),\right.\right. \\
& \left.\left.I\left(\tau_{n}, \omega\right), Q\left(\tau_{n}, \omega\right), R\left(\tau_{n}, \omega\right)\right)\right]=\mathbb{P}\left(\Omega_{n}\right) \mathbb{E} V\left(S \left(\tau_{n},\right.\right. \\
& \left.\omega), I\left(\tau_{n}, \omega\right), Q\left(\tau_{n}, \omega\right), R\left(\tau_{n}, \omega\right)\right) \geq \epsilon[(n-1-\ln n) \\
& \left.\wedge\left(\frac{1}{n}-1+\ln n\right)\right],
\end{aligned}
$$

where $1_{\Omega_{n}}$ represents the indicator function of $\Omega_{n}$. Letting $n \longrightarrow \infty$ in above inequality yields a contradiction that

$$
\infty>V\left(S_{0}, I_{0}, Q_{0}, R_{0}\right)+G T=\infty .
$$

Therefore, $\tau_{\infty}=\infty$ a.s. and this completes the proof.

\section{Stationary Distribution and Ergodicity}

In the field of infectious diseases, the persistence and extinction of diseases are an important research issue. In this section, we will show that system (2) has an ergodic stationary distribution under certain condition.

Theorem 6. Assume that

$$
R_{0}^{S}:=\frac{\beta \Lambda}{\left(\mu+\sigma_{1}^{2} / 2\right)\left(\gamma+\delta+\mu+\mu_{1}+\sigma_{2}^{2} / 2\right)}>1,
$$

then system (2) has a unique stationary distribution $\pi(\cdot)$ and it has the ergodic property.

Proof. By Theorem 5, we have obtained that, for any initial value $\left(S_{0}, I_{0}, Q_{0}, R_{0}\right) \in \mathbb{R}_{+}^{4}$, there is a unique global solution $(S, I, Q, R) \in \mathbb{R}_{+}^{4}$.

The diffusion matrix of system (2) is given by

$$
A=\left(\begin{array}{cccc}
\sigma_{1}^{2} S^{2} & 0 & 0 & 0 \\
0 & \sigma_{2}^{2} I^{2} & 0 & 0 \\
0 & 0 & \sigma_{3}^{2} Q^{2} & 0 \\
0 & 0 & 0 & \sigma_{4}^{2} R^{2}
\end{array}\right)
$$

Choose $M=\min _{(S, I, Q, R) \in \bar{D}_{\sigma} \subset E_{d}}\left\{\sigma_{1}^{2} S^{2}, \sigma_{2}^{2} I^{2}, \sigma_{3}^{2} Q^{2}, \sigma_{4}^{2} R^{2}\right\}$, where $E_{d}=: \mathbb{R}_{+}^{4}$ and $D_{\sigma}$ is a bounded domain in $E_{d}$, which does not contain the origin. For $\xi=\left(\xi_{1}, \xi_{2}, \xi_{3}, \xi_{4}\right) \in \mathbb{R}^{4}$, we have that

$$
\begin{aligned}
& \sum_{i, j=1}^{4} a_{i j}(S, I, Q, R) \xi_{i} \xi_{j}= \sigma_{1}^{2} S^{2} \xi_{1}^{2}+\sigma_{2}^{2} I^{2} \xi_{2}^{2}+\sigma_{3}^{2} Q^{2} \xi_{3}^{2} \\
&+ \sigma_{4}^{2} R^{2} \xi_{4}^{2} \geq M|\xi|^{2}, \\
&(S, I, Q, R) \in \bar{D}_{\sigma} .
\end{aligned}
$$

Then the condition $\widetilde{A_{1}}$ holds.

Denote

$$
\begin{aligned}
\eta & =\left(\mu+\frac{\sigma_{1}^{2}}{2}\right)^{1 / 2} \\
& \cdot\left[\left(\frac{\beta \Lambda}{\gamma+\delta+\mu+\mu_{1}+\sigma_{2}^{2} / 2}\right)^{1 / 2}-\left(\mu+\frac{\sigma_{1}^{2}}{2}\right)^{1 / 2}\right] .
\end{aligned}
$$

From the assumption $R_{0}^{S}>1$, we know that $\eta$ is positive. Construct a function $\widehat{V}: \mathbb{R}_{+}^{4} \longrightarrow \mathbb{R}$ in the following form:

$$
\begin{aligned}
\widehat{V}(S, I, Q, R)= & M(-\ln S-c \ln I) \\
& +\frac{1}{\rho+1}(S+I+Q+R)^{\rho+1}-\ln S \\
& -\ln Q-\ln R+(S+I+Q+R) \\
:= & M V_{1}+V_{2}+V_{3}+V_{4}+V_{5}+V_{6},
\end{aligned}
$$

where

$$
\begin{aligned}
& c=\frac{\mu+\sigma_{1}^{2} / 2}{\gamma+\delta+\mu+\mu_{1}+\sigma_{2}^{2} / 2}, \\
& 0<\rho<\frac{2 \mu}{\sigma_{1}^{2} \vee \sigma_{2}^{2} \vee \sigma_{3}^{2} \vee \sigma_{4}^{2}}
\end{aligned}
$$

and $M$ is positive constant which satisfying the following condition:

$$
-2 M \eta+\Lambda+B+3 \mu+\kappa+\mu_{2}+\frac{\sigma_{1}^{2}+\sigma_{3}^{2}+\sigma_{4}^{2}}{2} \leq-2,
$$

where $B$ is determined later. It is evident that

$$
\liminf _{k \rightarrow \infty,(S, I, Q, R) \in \mathbb{R}_{+}^{4} \backslash U_{k}} \widehat{V}(S, I, Q, R)=+\infty,
$$

where $U_{k}=(1 / k, k) \times(1 / k, k) \times(1 / k, k) \times(1 / k, k)$. In addition, $\widehat{V}(S, I, Q, R)$ is a continuous function. Hence $\widehat{V}(S, I, Q, R)$ has a minimum point $\left(\bar{S}_{0}, \bar{I}_{0}, \bar{Q}_{0}, \bar{R}_{0}\right)$ in the interior of $\mathbb{R}_{+}^{4}$. Then we define a nonnegative $C^{2}$-function $V: \mathbb{R}_{+}^{4} \longrightarrow \mathbb{R}_{+}$as follows:

$$
V(S, I, Q, R)=\widehat{V}(S, I, Q, R)-\widehat{V}\left(\bar{S}_{0}, \bar{I}_{0}, \bar{Q}_{0}, \bar{R}_{0}\right) .
$$


Making use of Itô's formula, we have

$$
\begin{aligned}
L V_{1}= & -\left(\frac{\Lambda}{S}+\frac{c \beta S}{1+\alpha I}\right)+\frac{\beta I}{1+\alpha I}+\mu+\frac{\sigma_{1}^{2}}{2} \\
& +c\left[\left(\gamma+\delta+\mu+\mu_{1}\right)+\frac{\sigma_{2}^{2}}{2}\right] .
\end{aligned}
$$

Utilizing the inequality $a+b \geq 2 \sqrt{a b}, a, b>0$, yields

$$
\begin{aligned}
& L V_{1} \leq-2\left(\frac{c \beta \Lambda}{1+\alpha I}\right)^{1 / 2}+\frac{\beta I}{1+\alpha I}+\mu+\frac{\sigma_{1}^{2}}{2}+c(\gamma+\delta \\
& \left.+\mu+\mu_{1}+\frac{\sigma_{2}^{2}}{2}\right) \\
& \quad=-2\left[\frac{\beta \Lambda\left(\mu+\sigma_{1}^{2} / 2\right)}{\left(\gamma+\delta+\mu+\mu_{1}+\sigma_{2}^{2} / 2\right)(1+\alpha I)}\right]^{1 / 2} \\
& +2\left(\mu+\frac{\sigma_{1}^{2}}{2}\right)+\frac{\beta I}{1+\alpha I}=-2\left(\mu+\frac{\sigma_{1}^{2}}{2}\right)^{1 / 2} \\
& \quad\left\{\left[\frac{\beta \Lambda}{\left(\gamma+\delta+\mu+\mu_{1}+\sigma_{2}^{2} / 2\right)(1+\alpha I)}\right]^{1 / 2}\right. \\
& \left.\quad-\left(\mu+\frac{\sigma_{1}^{2}}{2}\right)^{1 / 2}\right\}+\frac{\beta I}{1+\alpha I} .
\end{aligned}
$$

Meanwhile,

$$
\begin{aligned}
L V_{2} & =(S+I+Q+R)^{\rho} \\
\cdot & {\left[\Lambda-\mu S-\left(\mu+\mu_{1}\right) I-\left(\mu+\mu_{2}\right) Q-\mu R\right]+\frac{1}{2} } \\
\cdot & \rho(S+I+Q+R)^{\rho-1} \\
\times & \left(\sigma_{1}^{2} S^{2}+\sigma_{2}^{2} I^{2}+\sigma_{3}^{2} Q^{2}+\sigma_{4}^{2} R^{2}\right) \\
& \leq(S+I+Q+R)^{\rho}[\Lambda-\mu(S+I+Q+R)]+\frac{1}{2} \\
\cdot & \rho(S+I+Q+R)^{\rho+1}\left(\sigma_{1}^{2} \vee \sigma_{2}^{2} \vee \sigma_{3}^{2} \vee \sigma_{4}^{2}\right) \\
& =\Lambda(S+I+Q+R)^{\rho} \\
& -\left[\mu-\frac{1}{2} \rho\left(\sigma_{1}^{2} \vee \sigma_{2}^{2} \vee \sigma_{3}^{2} \vee \sigma_{4}^{2}\right)\right](S+I+Q+R)^{\rho+1} \\
& \leq B-\frac{1}{2}\left[\mu-\frac{1}{2} \rho\left(\sigma_{1}^{2} \vee \sigma_{2}^{2} \vee \sigma_{3}^{2} \vee \sigma_{4}^{2}\right)\right] \\
\cdot & (S+I+Q+R)^{\rho+1} \leq B \\
- & \frac{1}{2}\left[\mu-\frac{1}{2} \rho\left(\sigma_{1}^{2} \vee \sigma_{2}^{2} \vee \sigma_{3}^{2} \vee \sigma_{4}^{2}\right)\right] \\
& {\left[I^{\rho+1}+Q Q^{\rho+1}+R^{\rho+1}\right), }
\end{aligned}
$$

where

$$
\begin{aligned}
B= & \sup _{(S, I, Q, R) \in \mathbb{R}_{+}^{4}}\left\{\Lambda(S+I+Q+R)^{\rho}\right. \\
& -\frac{1}{2}\left[\mu-\frac{1}{2} \rho\left(\sigma_{1}^{2} \vee \sigma_{2}^{2} \vee \sigma_{3}^{2} \vee \sigma_{4}^{2}\right)\right] \\
& \left.\cdot(S+I+Q+R)^{\rho+1}\right\}<\infty .
\end{aligned}
$$

We can also obtain that

$$
\begin{aligned}
& L V_{3}=-\frac{\Lambda}{S}+\frac{\beta I}{1+\alpha I}+\mu+\frac{\sigma_{1}^{2}}{2}, \\
& L V_{4}=-\frac{\delta I}{Q}+\left(\kappa+\mu+\mu_{2}\right)+\frac{\sigma_{3}^{2}}{2}, \\
& L V_{5}=-\frac{\gamma I}{R}-\frac{\kappa Q}{R}+\mu+\frac{\sigma_{4}^{2}}{2}
\end{aligned}
$$

and

$$
L V_{6}=\Lambda-\mu(S+I+Q+R)-\mu_{1} I-\mu_{2} Q \leq \Lambda .
$$

Hence by (30), (31), (33), (34), (35), and (36), we can deduce that

$$
\begin{aligned}
L V & \leq-2 M\left(\mu+\frac{\sigma_{1}^{2}}{2}\right)^{1 / 2} \\
& .\left\{\left[\frac{\beta \Lambda}{\left(\gamma+\delta+\mu+\mu_{1}+\sigma_{2}^{2} / 2\right)(1+\alpha I)}\right]^{1 / 2}\right. \\
& \left.-\left(\mu+\frac{\sigma_{1}^{2}}{2}\right)^{1 / 2}\right\}+\frac{(M+1) \beta I}{1+\alpha I}-\frac{1}{2}[\mu \\
& \left.-\frac{1}{2} \rho\left(\sigma_{1}^{2} \vee \sigma_{2}^{2} \vee \sigma_{3}^{2} \vee \sigma_{4}^{2}\right)\right]\left(S^{\rho+1}+I^{\rho+1}+Q^{\rho+1}\right. \\
& \left.+R^{\rho+1}\right)-\frac{\Lambda}{S}-\frac{\delta I}{Q}-\frac{\gamma I}{R}-\frac{\kappa Q}{R}+\Lambda+B+3 \mu+\kappa \\
& +\mu_{2}+\frac{\sigma_{1}^{2}+\sigma_{3}^{2}+\sigma_{4}^{2}}{2} .
\end{aligned}
$$

Next, we will construct a compact subset $D$ such that the condition $A_{2}$ in Lemma 3 holds. Thus, we define the following bounded subset:

$$
\begin{aligned}
D & =\left\{\epsilon_{1} \leq S \leq \frac{1}{\epsilon_{1}}, \epsilon_{1} \leq I \leq \frac{1}{\epsilon_{1}}, \epsilon_{2} \leq Q \leq \frac{1}{\epsilon_{2}}, \epsilon_{2} \leq R\right. \\
& \left.\leq \frac{1}{\epsilon_{2}}\right\},
\end{aligned}
$$


where $\epsilon_{1}, \epsilon_{2}>0$ are small enough and satisfy the following conditions:

$$
\begin{aligned}
- & 2 M \tilde{\eta}+(M+1) \beta \epsilon_{1}+\Lambda+B+3 \mu+\kappa+\mu_{2} \\
& +\frac{\sigma_{1}^{2}+\sigma_{3}^{2}+\sigma_{4}^{2}}{2} \leq-1, \\
- & \frac{\Lambda}{\epsilon_{1}}+E \leq-1, \\
- & \frac{\delta}{\epsilon_{1}}+E \leq-1, \\
- & \frac{\gamma}{\epsilon_{1}}+E \leq-1, \\
- & \frac{1}{2}\left[\mu-\frac{1}{2} \rho\left(\sigma_{1}^{2} \vee \sigma_{2}^{2} \vee \sigma_{3}^{2} \vee \sigma_{4}^{2}\right)\right] \frac{1}{\epsilon_{1}^{\rho+1}}+E \leq-1, \\
- & \frac{1}{2}\left[\mu-\frac{1}{2} \rho\left(\sigma_{1}^{2} \vee \sigma_{2}^{2} \vee \sigma_{3}^{2} \vee \sigma_{4}^{2}\right)\right] \frac{1}{\epsilon_{2}^{\rho+1}}+E \leq-1,
\end{aligned}
$$

where

$$
\begin{aligned}
\widetilde{\eta}: & =\left(\mu+\frac{\sigma_{1}^{2}}{2}\right)^{1 / 2} \\
& \cdot\left\{\left[\frac{\beta \Lambda}{\left(\gamma+\delta+\mu+\mu_{1}+\sigma_{2}^{2} / 2\right)\left(1+\alpha \epsilon_{1}\right)}\right]^{1 / 2}\right. \\
& \left.-\left(\mu+\frac{\sigma_{1}^{2}}{2}\right)^{1 / 2}\right\} .
\end{aligned}
$$

Note that, for sufficiently small $\epsilon_{1},(39)$ is valid due to inequality (26).

It can been easily seen that $\mathbb{R}_{+}^{4} \backslash D$ is divided into eight domains, which can be denoted as

$$
\begin{aligned}
D_{1} & =\left\{(S, I, Q, R) \in \mathbb{R}_{+}^{4}, 0<S<\epsilon_{1}\right\}, \\
D_{2} & =\left\{(S, I, Q, R) \in \mathbb{R}_{+}^{4}, 0<I<\epsilon_{1}\right\}, \\
D_{3} & =\left\{(S, I, Q, R) \in \mathbb{R}_{+}^{4}, S \geq \epsilon_{1}, I \geq \epsilon_{1}, 0<Q<\epsilon_{2}\right\}, \\
D_{4} & =\left\{(S, I, Q, R) \in \mathbb{R}_{+}^{4}, I \geq \epsilon_{1}, 0<R<\epsilon_{2}\right\}, \\
D_{5} & =\left\{(S, I, Q, R) \in \mathbb{R}_{+}^{4}, S>\frac{1}{\epsilon_{1}}\right\}, \\
D_{6} & =\left\{(S, I, Q, R) \in \mathbb{R}_{+}^{4}, I>\frac{1}{\epsilon_{1}}\right\}, \\
D_{7} & =\left\{(S, I, Q, R) \in \mathbb{R}_{+}^{4}, Q>\frac{1}{\epsilon_{2}}\right\}, \\
D_{8} & =\left\{(S, I, Q, R) \in \mathbb{R}_{+}^{4}, R>\frac{1}{\epsilon_{2}}\right\} .
\end{aligned}
$$

Consequently, we consider the condition $A_{2}$ from the following eight cases.

Case 1. If $(S, I, Q, R) \in D_{1}$, from (37), we can get that

$$
\begin{aligned}
L V \leq & -\frac{\Lambda}{S}+2 M\left(\mu+\frac{\sigma_{1}^{2}}{2}\right)+\frac{(M+1) \beta}{\alpha}+\Lambda+B \\
& +3 \mu+\kappa+\mu_{2}+\frac{\sigma_{1}^{2}+\sigma_{3}^{2}+\sigma_{4}^{2}}{2}=-\frac{\Lambda}{S}+E \\
\leq & -\frac{\Lambda}{\epsilon_{1}}+E
\end{aligned}
$$

where

$$
\begin{aligned}
E= & 2 M\left(\mu+\frac{\sigma_{1}^{2}}{2}\right)+\frac{(M+1) \beta}{\alpha}+\Lambda+B+3 \mu+\kappa \\
& +\mu_{2}+\frac{\sigma_{1}^{2}+\sigma_{3}^{2}+\sigma_{4}^{2}}{2} .
\end{aligned}
$$

Then it infers from (40) that

$$
L V \leq-1
$$

Case 2. If $(S, I, Q, R) \in D_{2}$, we obtain that

$$
\begin{aligned}
L V & \leq-2 M\left(\mu+\frac{\sigma_{1}^{2}}{2}\right)^{1 / 2} \\
& .\left\{\left[\frac{\beta \Lambda}{\left(\gamma+\delta+\mu+\mu_{1}+\sigma_{2}^{2} / 2\right)(1+\alpha I)}\right]^{1 / 2}\right. \\
& \left.-\left(\mu+\frac{\sigma_{1}^{2}}{2}\right)^{1 / 2}\right\}+(M+1) \beta I+\Lambda+B+3 \mu+\kappa \\
& +\mu_{2}+\frac{\sigma_{1}^{2}+\sigma_{3}^{2}+\sigma_{4}^{2}}{2} \leq-2 M \tilde{\eta}+(M+1) \beta \epsilon_{1}+\Lambda \\
& +B+3 \mu+\kappa+\mu_{2}+\frac{\sigma_{1}^{2}+\sigma_{3}^{2}+\sigma_{4}^{2}}{2} .
\end{aligned}
$$

In view of (39), we have that

$$
L V \leq-1
$$

Case 3. If $(S, I, Q, R) \in D_{3}$, we have

$$
\begin{aligned}
L V \leq & -\frac{\delta I}{Q}+2 M\left(\mu+\frac{\sigma_{1}^{2}}{2}\right)+\frac{(M+1) \beta}{\alpha}+\Lambda+B \\
& +3 \mu+\kappa+\mu_{2}+\frac{\sigma_{1}^{2}+\sigma_{3}^{2}+\sigma_{4}^{2}}{2} \leq-\frac{\delta \epsilon_{1}}{\epsilon_{2}}+E .
\end{aligned}
$$

Choosing

$$
\epsilon_{2}=\epsilon_{1}^{2}
$$


and combing with (41), we obtain that

$$
L V \leq-\frac{\delta}{\epsilon_{1}}+E \leq-1 .
$$

Case 4. If $(S, I, Q, R) \in D_{4}$, using the similar technique in Case 3 and (42), we have

$$
\begin{aligned}
L V \leq & -\frac{\gamma I}{R}+2 M\left(\mu+\frac{\sigma_{1}^{2}}{2}\right)+\frac{(M+1) \beta}{\alpha}+\Lambda+B \\
& +3 \mu+\kappa+\mu_{2}+\frac{\sigma_{1}^{2}+\sigma_{3}^{2}+\sigma_{4}^{2}}{2} \leq-\frac{\gamma \epsilon_{1}}{\epsilon_{2}}+E \\
= & -\frac{\gamma}{\epsilon_{1}}+E \leq-1 .
\end{aligned}
$$

Case 5. If $(S, I, Q, R) \in D_{5}$, we can derive that

$$
\begin{aligned}
L V \leq & -\frac{1}{2}\left[\mu-\frac{1}{2} \rho\left(\sigma_{1}^{2} \vee \sigma_{2}^{2} \vee \sigma_{3}^{2} \vee \sigma_{4}^{2}\right)\right] S^{\rho+1} \\
& +2 M\left(\mu+\frac{\sigma_{1}^{2}}{2}\right)+\frac{(M+1) \beta}{\alpha}+\Lambda+B+3 \mu \\
& +\kappa+\mu_{2}+\frac{\sigma_{1}^{2}+\sigma_{3}^{2}+\sigma_{4}^{2}}{2} \\
\leq & -\frac{1}{2}\left[\mu-\frac{1}{2} \rho\left(\sigma_{1}^{2} \vee \sigma_{2}^{2} \vee \sigma_{3}^{2} \vee \sigma_{4}^{2}\right)\right] \frac{1}{\epsilon_{1}^{\rho+1}}+E .
\end{aligned}
$$

By (43), we can conclude that $L V \leq-1$ on $D_{5}$.

Case 6. If $(S, I, Q, R) \in D_{6}$, using the analogous estimation in Case 5 and (43), we have

$$
\begin{aligned}
L V \leq & -\frac{1}{2}\left[\mu-\frac{1}{2} \rho\left(\sigma_{1}^{2} \vee \sigma_{2}^{2} \vee \sigma_{3}^{2} \vee \sigma_{4}^{2}\right)\right] I^{\rho+1} \\
& +2 M\left(\mu+\frac{\sigma_{1}^{2}}{2}\right)+\frac{(M+1) \beta}{\alpha}+\Lambda+B+3 \mu \\
& +\kappa+\mu_{2}+\frac{\sigma_{1}^{2}+\sigma_{3}^{2}+\sigma_{4}^{2}}{2} \\
\leq & -\frac{1}{2}\left[\mu-\frac{1}{2} \rho\left(\sigma_{1}^{2} \vee \sigma_{2}^{2} \vee \sigma_{3}^{2} \vee \sigma_{4}^{2}\right)\right] \frac{1}{\epsilon_{1}^{\rho+1}}+E \\
\leq & -1 .
\end{aligned}
$$

Case 7. If $(S, I, Q, R) \in D_{7}$, it infers that

$$
\begin{aligned}
L V \leq & -\frac{1}{2}\left[\mu-\frac{1}{2} \rho\left(\sigma_{1}^{2} \vee \sigma_{2}^{2} \vee \sigma_{3}^{2} \vee \sigma_{4}^{2}\right)\right] Q^{\rho+1} \\
& +2 M\left(\mu+\frac{\sigma_{1}^{2}}{2}\right)+\frac{(M+1) \beta}{\alpha}+\Lambda+B+3 \mu \\
& +\kappa+\mu_{2}+\frac{\sigma_{1}^{2}+\sigma_{3}^{2}+\sigma_{4}^{2}}{2} \\
\leq & -\frac{1}{2}\left[\mu-\frac{1}{2} \rho\left(\sigma_{1}^{2} \vee \sigma_{2}^{2} \vee \sigma_{3}^{2} \vee \sigma_{4}^{2}\right)\right] \frac{1}{\epsilon_{2}^{\rho+1}}+E .
\end{aligned}
$$

By (44), we can conclude that $L V \leq-1$ on $D_{7}$.

Case 8. At last, if $(S, I, Q, R) \in D_{8}$, by (44), we have

$$
\begin{aligned}
L V \leq & -\frac{1}{2}\left[\mu-\frac{1}{2} \rho\left(\sigma_{1}^{2} \vee \sigma_{2}^{2} \vee \sigma_{3}^{2} \vee \sigma_{4}^{2}\right)\right] R^{\rho+1} \\
& +2 M\left(\mu+\frac{\sigma_{1}^{2}}{2}\right)+\frac{(M+1) \beta}{\alpha}+\Lambda+B+3 \mu \\
& +\kappa+\mu_{2}+\frac{\sigma_{1}^{2}+\sigma_{3}^{2}+\sigma_{4}^{2}}{2} \\
\leq & -\frac{1}{2}\left[\mu-\frac{1}{2} \rho\left(\sigma_{1}^{2} \vee \sigma_{2}^{2} \vee \sigma_{3}^{2} \vee \sigma_{4}^{2}\right)\right] \frac{1}{\epsilon_{2}^{\rho+1}}+E \\
\leq & -1 .
\end{aligned}
$$

According to the discussion of the above eight cases, we obtain that

$$
L V \leq-1 \quad \forall(S, I, Q, R) \in \mathbb{R}_{+}^{4} \backslash D .
$$

Therefore, condition $\widetilde{A}_{2}$ is satisfied, and we can obtain that system (2) is ergodic and has a unique stationary distribution. The proof is complete.

Remark 7. Theorem 6 gives the sufficient condition of the existence of stationary distribution for the solution of (2). In epidemiology, this means that the stochastic model (2) oscillates around the endemic equilibrium of the corresponding deterministic model and implies the persistence of the disease a.s. under some condition.

\section{Extinction of the Disease}

In this section, we will establish the sufficient conditions for the extinction of the disease in the system (2), which is another important issue in epidemiology. For simplicity, we define

$$
\langle x(t)\rangle=\frac{1}{t} \int_{0}^{t} x(u) d u .
$$

Lemma 8. Let $(S(t), I(t), Q(t), R(t))$ be the solution of system (2) with any initial value $\left(S_{0}, I_{0}, Q_{0}, R_{0}\right) \in \mathbb{R}_{+}^{4}$. Then

$$
\lim _{t \rightarrow \infty} \frac{S(t)+I(t)+Q(t)+R(t)}{t}=0, \quad \text { a.s. }
$$

Moreover, if $\mu>\left(\sigma_{1}^{2} \vee \sigma_{2}^{2} \vee \sigma_{3}^{2} \vee \sigma_{4}^{2}\right) / 2$, then 


$$
\begin{aligned}
& \lim _{t \rightarrow \infty} \frac{1}{t} \int_{0}^{t} S(u) d B_{1}(u)=0, \\
& \lim _{t \rightarrow \infty} \frac{1}{t} \int_{0}^{t} I(u) d B_{2}(u)=0, \\
& \lim _{t \rightarrow \infty} \frac{1}{t} \int_{0}^{t} Q(u) d B_{3}(u)=0, \\
& \lim _{t \rightarrow \infty} \frac{1}{t} \int_{0}^{t} R(u) d B_{4}(u)=0,
\end{aligned}
$$$$
\text { a.s. }
$$

a.s.

Lemma 8 can be proved by using the same methods as that in Lemmas 2.1 and 2.2 of Zhao and Jiang [32], so we omit it here.

Define a constant as follows:

$$
\widehat{R}_{0}^{S}:=\frac{\beta \Lambda / \mu-\sigma_{2}^{2} / 2}{\delta+\gamma+\mu+\mu_{1}}=R_{0}-\frac{\sigma_{2}^{2}}{2\left(\delta+\gamma+\mu+\mu_{1}\right)},
$$

where $R_{0}=\beta \Lambda / \mu\left(\delta+\gamma+\mu+\mu_{1}\right)$ is the threshold value of the corresponding deterministic system for (2). Therefore, we obtain the following main result in this section.

Theorem 9. Assume that $\mu>\left(\sigma_{1}^{2} \vee \sigma_{2}^{2} \vee \sigma_{3}^{2} \vee \sigma_{4}^{2}\right) / 2$ and $(S(t), I(t), Q(t), R(t))$ is the solution of system (2) with any initial value $\left(S_{0}, I_{0}, Q_{0}, R_{0}\right) \in \mathbb{R}_{+}^{4}$. If $\widehat{R}_{0}^{S}<1$, then

$$
\lim _{t \rightarrow \infty} \frac{\ln I(t)}{t} \leq\left(\delta+\gamma+\mu+\mu_{1}\right)\left(\widehat{R}_{0}^{S}-1\right) \quad \text { a.s. }
$$

and

$$
\begin{gathered}
\lim _{t \longrightarrow \infty}\langle S(t)\rangle=\frac{\beta \Lambda}{\mu}, \\
\lim _{t \rightarrow \infty} Q(t)=0, \\
\lim _{t \rightarrow \infty} R(t)=0
\end{gathered}
$$

a.s.

Proof. By system (2), we have

$$
\begin{aligned}
& d(S(t)+I(t)+Q(t)+R(t))=[\Lambda \\
& \quad-\mu(S(t)+I(t)+Q(t)+R(t))-\mu_{1} I(t) \\
& \left.\quad-\mu_{2} Q(t)\right] d t+\sigma_{1} S(t) d B_{1}(t)+\sigma_{2} I(t) d B_{2}(t) \\
& \quad+\sigma_{3} Q(t) d B_{3}(t)+\sigma_{4} R(t) d B_{4}(t) .
\end{aligned}
$$

Integrating the both sides of (68) yields

$$
\begin{aligned}
& \frac{S(t)-S(0)}{t}+\frac{I(t)-I(0)}{t}+\frac{Q(t)-Q(0)}{t} \\
& \quad+\frac{R(t)-R(0)}{t} \\
& =\Lambda-\mu\langle S(t)\rangle-\left(\mu+\mu_{1}\right)\langle I(t)\rangle \\
& \quad-\left(\mu+\mu_{2}\right)\langle Q(t)\rangle-\mu\langle R(t)\rangle \\
& \quad+\frac{\sigma_{1}}{t} \int_{0}^{t} S(u) d B_{1}(u)+\frac{\sigma_{2}}{t} \int_{0}^{t} I(u) d B_{2}(u) \\
& \quad+\frac{\sigma_{3}}{t} \int_{0}^{t} Q(u) d B_{3}(u)+\frac{\sigma_{4}}{t} \int_{0}^{t} R(u) d B_{4}(u),
\end{aligned}
$$

then

$$
\begin{aligned}
\langle S(t)\rangle= & \frac{\Lambda}{\mu}-\frac{\mu+\mu_{1}}{\mu}\langle I(t)\rangle-\frac{\mu+\mu_{2}}{\mu}\langle Q(t)\rangle \\
& -\langle R(t)\rangle+\varphi(t),
\end{aligned}
$$

where $\varphi(t)$ is denoted as below:

$$
\begin{aligned}
& \varphi(t)=-\frac{1}{\mu}\left[\frac{S(t)-S(0)}{t}+\frac{I(t)-I(0)}{t}\right. \\
& +\frac{Q(t)-Q(0)}{t}+\frac{R(t)-R(0)}{t} \\
& -\frac{\sigma_{1}}{t} \int_{0}^{t} S(u) d B_{1}(u)-\frac{\sigma_{2}}{t} \int_{0}^{t} I(u) d B_{2}(u) \\
& \left.-\frac{\sigma_{3}}{t} \int_{0}^{t} Q(u) d B_{3}(u)-\frac{\sigma_{4}}{t} \int_{0}^{t} R(u) d B_{4}(u)\right] .
\end{aligned}
$$

Due to (62)-(64), we have

$$
\lim _{t \longrightarrow \infty} \varphi(t)=0 \quad \text { a.s. }
$$

By utilizing Itô's formula to the $I$ component of system (2), we have

$$
\begin{aligned}
d(\ln I)= & {\left[\frac{\beta S}{1+\alpha I}-\left(\delta+\gamma+\mu+\mu_{1}\right)-\frac{1}{2} \sigma_{2}^{2}\right] d t } \\
& +\sigma_{2} d B_{2}(t) \\
\leq & {\left[\beta S-\left(\delta+\gamma+\mu+\mu_{1}\right)-\frac{1}{2} \sigma_{2}^{2}\right] d t } \\
& +\sigma_{2} d B_{2}(t) .
\end{aligned}
$$

Integrating both sides of the above inequality from 0 to $t$ and dividing $t$, we obtain

$$
\begin{aligned}
\frac{\ln I(t)-\ln I(0)}{t} \leq & \beta\langle S(t)\rangle-\left(\delta+\gamma+\mu+\mu_{1}\right)-\frac{\sigma_{2}^{2}}{2} \\
& +\frac{\sigma_{2} B_{2}(t)}{t} .
\end{aligned}
$$


Substituting (70) into (74) leads to

$$
\begin{aligned}
\frac{\ln I(t)}{t} \leq & \frac{\beta \Lambda}{\mu}-\left(\delta+\gamma+\mu+\mu_{1}\right)-\frac{\sigma_{2}^{2}}{2}+\beta \varphi(t) \\
& +\frac{\sigma_{2} B_{2}(t)}{t}+\frac{\ln I(0)}{t} \\
= & \left(\delta+\gamma+\mu+\mu_{1}\right)\left(\widehat{R}_{0}^{S}-1\right)+\beta \varphi(t) \\
& +\frac{\sigma_{2} B_{2}(t)}{t}+\frac{\ln I(0)}{t} .
\end{aligned}
$$

Due to the fact that $\lim _{t \rightarrow \infty}\left(B_{2}(t) / t\right)=0$ a.s. and (72), by taking the superior limit on the both sides of (75), if $\widehat{R}_{0}^{S}<1$, then

$$
\limsup _{t \rightarrow \infty} \frac{\ln I(t)}{t} \leq\left(\delta+\gamma+\mu+\mu_{1}\right)\left(\widehat{R}_{0}^{S}-1\right)<0
$$

a.s.,

which infers that

$$
\lim _{t \rightarrow \infty} I(t)=0 \quad \text { a.s. }
$$

Hence, from (77) and the last two equations of (2), we have

$$
\begin{aligned}
& \lim _{t \longrightarrow \infty} Q(t)=0, \\
& \lim _{t \longrightarrow \infty} R(t)=0
\end{aligned}
$$

a.s.

At last, by (72), (77), and (78) and from (70), we obtain

$$
\lim _{t \rightarrow \infty}\langle S(t)\rangle=\frac{\beta \Lambda}{\mu} \quad \text { a.s. }
$$

This completes the proof.

\section{Numerical Simulations}

In this section, we give some numerical simulations in order to illustrate the theoretical results. For numerical simulation, by using Milstein's method [33], we obtain the following discretization transformation of system (2):

$$
\begin{aligned}
S_{j+1}= & S_{j}+\left[\Lambda-\frac{\beta S_{j} I_{j}}{1+\alpha I_{j}}-\mu S_{j}\right] \Delta t+\sigma_{1} S_{j} \sqrt{\Delta t} \varepsilon_{1, j} \\
& +\frac{\sigma_{1}^{2}}{2} S_{j}\left(\varepsilon_{1, j}^{2}-1\right) \Delta t, \\
I_{j+1}= & I_{j}+\left[\frac{\beta S_{j} I_{j}}{1+\alpha I_{j}}-\left(\delta+\gamma+\mu+\mu_{1}\right) I_{j}\right] \Delta t \\
& +\sigma_{2} I_{j} \sqrt{\Delta t} \varepsilon_{2, j}+\frac{\sigma_{2}^{2}}{2} I_{j}\left(\varepsilon_{2, j}^{2}-1\right) \Delta t, \\
Q_{j+1}= & Q_{j}+\left(\delta I_{j}-\left(\kappa+\mu+\mu_{2}\right) Q_{j}\right) \Delta t \\
& +\sigma_{3} Q_{j} \sqrt{\Delta t} \varepsilon_{3, j}+\frac{\sigma_{3}^{2}}{2} Q_{j}\left(\varepsilon_{3, j}^{2}-1\right) \Delta t, \\
R_{j+1}= & R_{j}+\left(\gamma I_{j}+\kappa Q_{j}-\mu R_{j}\right) \Delta t+\sigma_{4} R_{j} \sqrt{\Delta t} \varepsilon_{4, j} \\
& +\frac{\sigma_{4}^{2}}{2} R_{j}\left(\varepsilon_{4, j}^{2}-1\right) \Delta t,
\end{aligned}
$$

where $\Delta t>0, \sigma_{i}^{2}>0(i=1,2,3,4)$ are the intensities of the white noise, $\varepsilon_{i, j}(i=1,2,3,4, j=1,2, \ldots, n)$ are independent Gaussian random variables which follow $N(0,1)$. In order to verify the persistence and extinction results, we choose the parameters in system (80) by $\beta=0.3, \mu=\mu_{1}=$ $0.1, \mu_{2}=\gamma=\delta=0.2, \kappa=0.1, \alpha=0.5, \sigma_{1}=\sigma_{3}=0.05$, and $\sigma_{4}=0.1$.

Firstly, we start our numerical simulation with $\Lambda=$ $0.3, \sigma_{2}=0.1$ and starting from the initial point $(2,0.5,0.5,1)$. Direct calculation leads to $R_{0}^{S}:=\beta \Lambda /(\mu+$ $\left.\sigma_{1}^{2} / 2\right)\left(\gamma+\delta+\mu+\mu_{1}+\sigma_{2}^{2} / 2\right) \approx 1.4692>1$. Thus, from Theorem 6 , we can conclude that system (2) has a unique ergodic stationary distribution which leads to the stochastically persistence of the disease. Figure 1 confirms this.

Next, we increase the stochastic intensity of $I$ to $\sigma_{2}=0.4$ and choose $\Lambda=0.22$. In this case, $\mu>\left(\sigma_{1}^{2} \vee \sigma_{2}^{2} \vee \sigma_{3}^{2} \vee \sigma_{4}^{2}\right) / 2$ and $\widehat{R}_{0}^{S}:=R_{0}-\sigma_{2}^{2} / 2\left(\delta+\gamma+\mu+\mu_{1}\right) \approx 0.9667<1<$ $R_{0}$. According to Theorem 9, we obtain that system (2) has disease dies out with probability 1 , and Figure 2 illustrates this.

\section{Conclusion and Discussion}

In this paper, we consider a stochastic SIQR epidemic model with saturated incidence rate. By constructing proper Lyapunov function, we establish the existence and uniqueness of solutions of system (2). Furthermore, we obtain the existence of ergodic stationary distribution of the solution to (2) when $R_{0}^{S}>1$. Meanwhile, we establish sufficient conditions for the extinction of the disease as well, that is, $\mu>\left(\sigma_{1}^{2} \vee \sigma_{2}^{2} \vee \sigma_{3}^{2} \vee\right.$ $\left.\sigma_{4}^{2}\right) / 2$ and $\widehat{R}_{0}^{S}<1$.

It is worth further studying the following two topics. On the one hand, noting that $R_{0}^{S} \neq \widehat{R}_{0}^{S}$, it is of great interest 

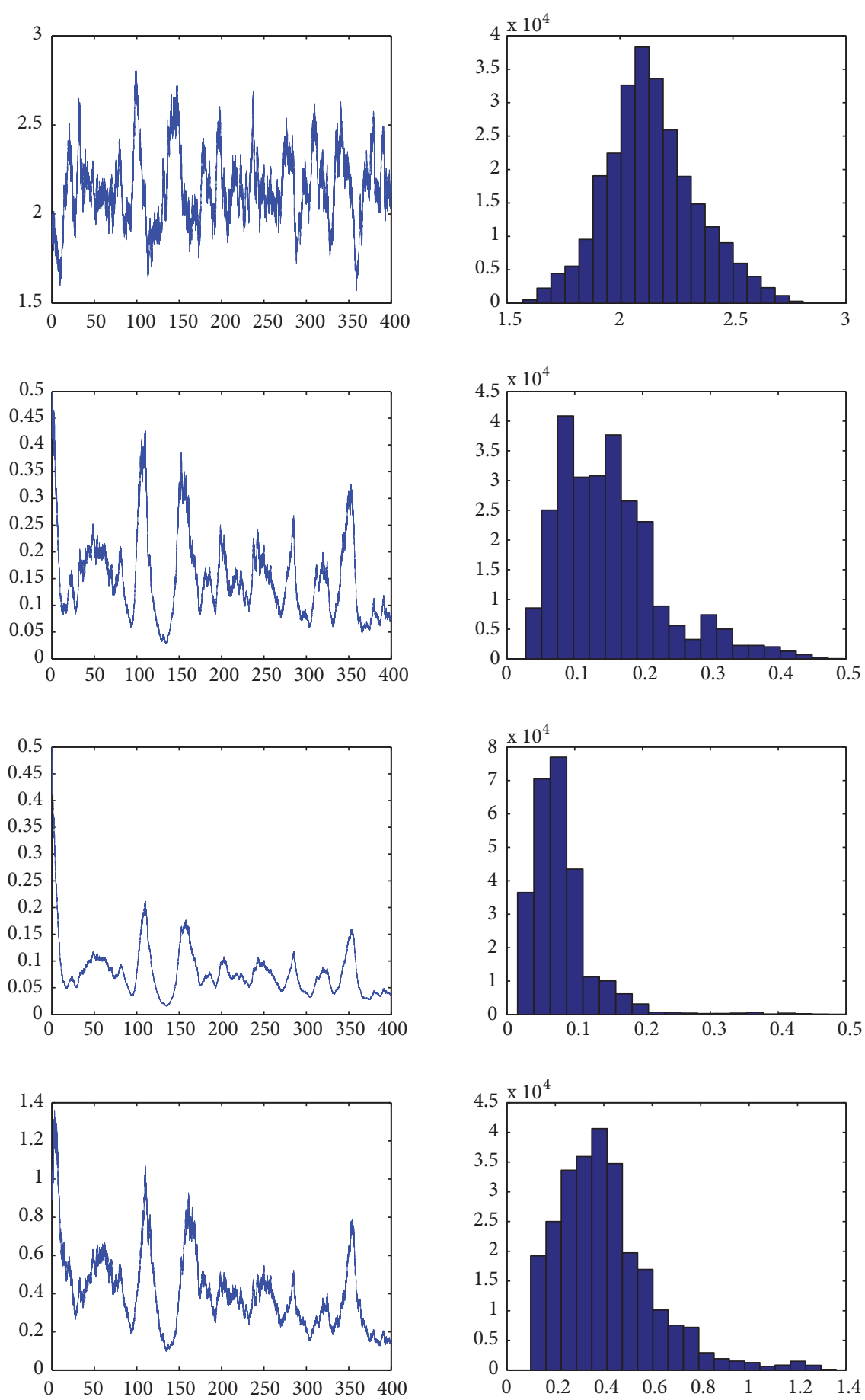

Figure 1: The left column shows the solutions of stochastic system (2) under the noise intensities $\sigma_{1}=\sigma_{3}=0.05, \sigma_{2}=0.4, \sigma_{4}=0.1$. The right column displays the histogram of the probability density function of $S(t), I(t), Q(t), R(t)$.

that whether can we promote the sufficient conditions for the extinction of the disease and get the threshold value for the extinction and persistence of the disease? On the other hand, we can propose SIQR epidemic model with some other types of random perturbations, such as telephone noise which can cause the population system to switch among different environmental regimes (see, e.g., [34-37]). In the literatures, it is common to use the continuous-time Markov chain with values in a finite state space to describe the random switching of environmental regimes. We will carry out these studies in the future.

\section{Data Availability}

No data were used to support this study. 

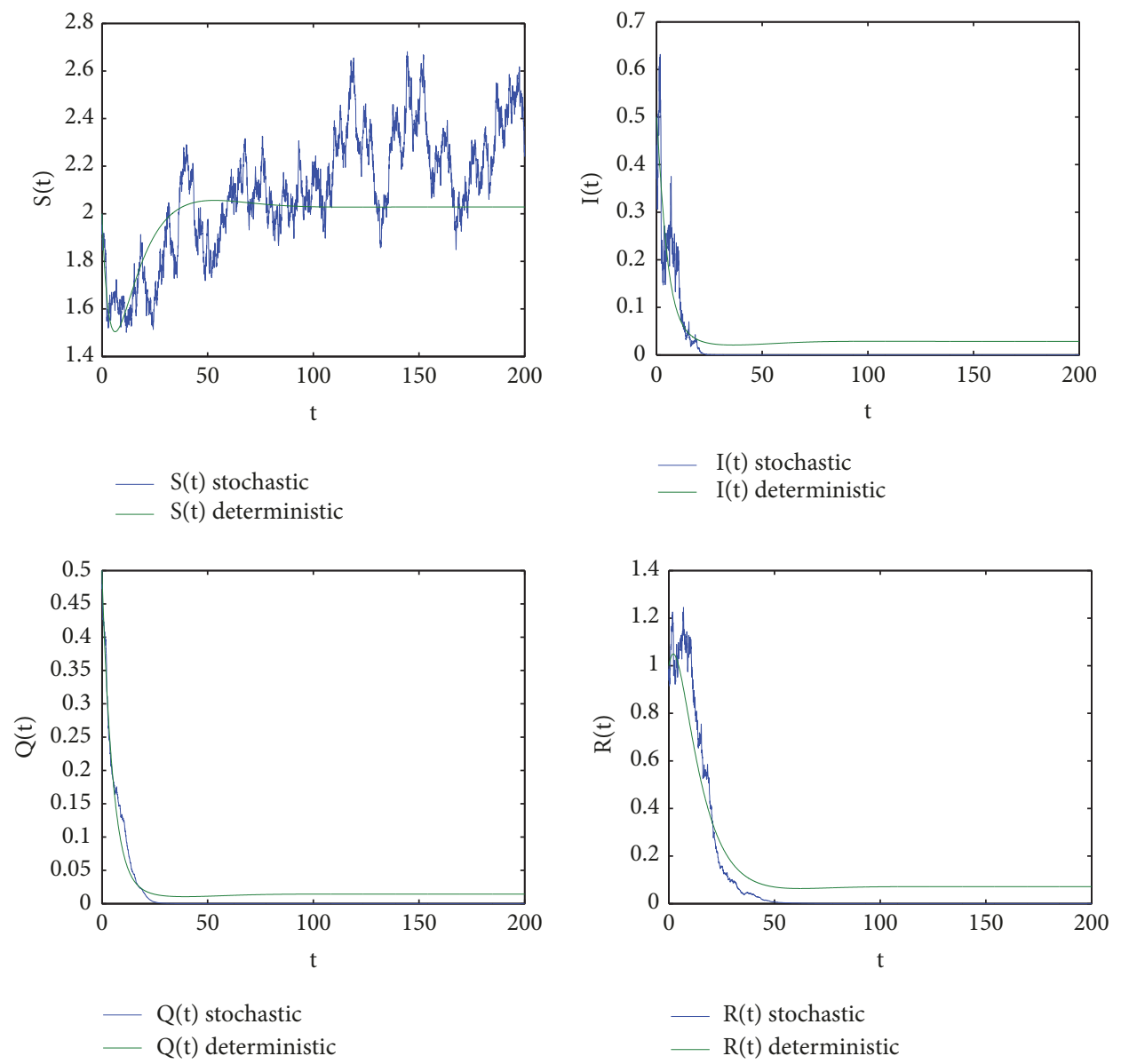

FIgURE 2: Numerical simulation for system (2) with $\sigma_{1}=\sigma_{3}=0.05, \sigma_{2}=\sigma_{4}=0.1$.

\section{Conflicts of Interest}

The authors declare that they have no conflicts of interest.

\section{Acknowledgments}

This work is supported by the National Natural Science Foundation of China (no. 11761015), Anhui Provincial Natural Science Foundation (no. 1708085QA13), the Natural Science Foundation of Anhui Provincial Education Department (nos. KJ2016A517; KJ2018A0578), the Outstanding Young Talents Project of Anhui Provincial Universities (no. gxgwfx2018081), and the Teaching Team of Chizhou University (no. 2018XJXTD03).

\section{References}

[1] W. O. Kermack and A. G. McKendrick, "A contribution to the mathematical theory of epidemics," Proceedings of the Royal Society of London. Series A, vol. 115, no. 772, pp. 700-721, 1927.

[2] E. Beretta, T. Hara, W. Ma, and Y. Takeuchi, "Global asymptotic stability of an SIR epidemic model with distributed time delay," Nonlinear Analysis, vol. 47, no. 6, pp. 4107-4115, 2011.

[3] E. Beretta and Y. Takeuchi, "Global stability of an SIR epidemic model with time delays," Journal of Mathematical Biology, vol. 33, no. 3, pp. 250-260, 1995.
[4] K. L. Cooke and P. van den Driessche, "Analysis of an SEIRS epidemic model with two delays," Journal of Mathematical Biology, vol. 35, no. 2, pp. 240-260, 1995.

[5] K. Cooke, P. van den Driessche, and X. Zou, "Interaction of maturation delay and nonlinear birth in population and epidemic models," Journal of Mathematical Biology, vol. 39, no. 4, pp. 332-352, 1999.

[6] H. L. Smith, "Subharmonic bifurcation in an SIR epidemic model," Journal of Mathematical Biology, vol. 17, no. 2, pp. 163$177,1983$.

[7] G. Lan, Z. Chen, C. Wei, and S. Zhang, "Stationary distribution of a stochastic SIQR epidemic model with saturated incidence and degenerate diffusion," Physica A: Statistical Mechanics and its Applications, vol. 511, pp. 61-77, 2018.

[8] Z. Feng and H. R. Thieme, "Recurrent outbreaks of childhood diseases revisited: the impact of isolation," Mathematical Biosciences, vol. 128, no. 1-2, pp. 93-130, 1995.

[9] H. Joshi, R. Sharma, and G. Prajapati, "Global dynamics of an SIQR epidemic model with saturated incidence rate," Asian Journal of Mathematics and Computer Research, vol. 21, no. 4, pp. 156-166, 2017.

[10] L.-I. Wu and Z. L. Feng, "Homoclinic bifurcation in an SIQR model for childhood diseases," Journal of Differential Equations, vol. 168, no. 1, pp. 150-167, 2000.

[11] H. Herbert, Z. Ma, and S. Liao, "Effects of quarantine in six endemic models for infectious diseases," Mathematical Biosciences, vol. 180, no. 1-2, pp. 141-160, 2002. 
[12] N. Nirwani, V. Badshah, and R. Khandelwal, "Dynamical study of an SIQR model with saturated incidence rate," Nonlinear Analysis and Differential Equations, vol. 4, no. 1, pp. 43-50, 2016.

[13] Y. Cai, Y. Kang, M. Banerjee, and W. Wang, "A stochastic SIRS epidemic model with infectious force under intervention strategies," Journal of Differential Equations, vol. 259, no. 12, pp. 7463-7502, 2015.

[14] Z. Cao and S. Zhou, "Dynamical behaviors of a stochastic SIQR epidemic model with quarantine-adjusted incidence," Discrete Dynamics in Nature and Society, vol. 2018, Article ID 3693428, 13 pages, 2018.

[15] R. Z. Khasminskii and F. C. Klebaner, "Long term behavior of solutions of the Lotka-Volterra system under small random perturbations," The Annals of Applied Probability, vol. 11, no. 3, pp. 952-963, 2001.

[16] A. Lahrouz and L. Omari, "Extinction and stationary distribution of a stochastic SIRS epidemic model with non-linear incidence," Statistics \& Probability Letters, vol. 83, no. 4, pp. 960968, 2013.

[17] E. Allen, "Environmental variability and mean-reverting processes," Discrete and Continuous Dynamical Systems - Series B, vol. 21, no. 7, pp. 2073-2089, 2016.

[18] Y. Cai, J. Jiao, Z. Gui, Y. Liu, and W. Wang, "Environmental variability in a stochastic epidemic model," Applied Mathematics and Computation, vol. 329, pp. 210-226, 2018.

[19] D. Li, J. Cui, M. Liu, and S. Liu, "The evolutionary dynamics of stochastic epidemic model with nonlinear incidence rate," Bulletin of Mathematical Biology, vol. 77, no. 9, pp. 1705-1743, 2015.

[20] M. Liu and K. Wang, "Dynamics of a two-prey one-predator system in random environments," Journal of Nonlinear Science, vol. 23, no. 5, pp. 751-775, 2013.

[21] Q. Liu and D. Jiang, "Stationary distribution and extinction of a stochastic SIR model with nonlinear perturbation," Applied Mathematics Letters, vol. 73, pp. 8-15, 2017.

[22] Q. Liu, D. Jiang, and N. Shi, “Threshold behavior in a stochastic SIQR epidemic model with standard incidence and regime switching," Applied Mathematics and Computation, vol. 316, pp. 310-325, 2018.

[23] Q. Liu, D. Jiang, N. Shi, T. Hayat, and B. Ahmad, "Stationary distribution and extinction of a stochastic SEIR epidemic model with standard incidence," Physica A: Statistical Mechanics and its Applications, vol. 476, pp. 58-69, 2017.

[24] Q. Liu, D. Jiang, N. Shi, T. Hayat, and A. Alsaedi, "Stationary distribution and extinction of a stochastic SIRS epidemic model with standard incidence," Physica A: Statistical Mechanics and its Applications, vol. 469, pp. 510-517, 2017.

[25] Y. Xu, X. Jin, and H. Zhang, "Parallel logic gates in synthetic gene networks induced by non-Gaussian noise," Physical Review E, vol. 88, no. 5, Article ID 052721, 2013.

[26] Q. Yang and X. Mao, "Extinction and recurrence of multigroup SEIR epidemic models with stochastic perturbations," Nonlinear Analysis: Real World Applications, vol. 14, no. 3, pp. 1434-1456, 2013.

[27] Y. Cai, Y. Kang, and W. Wang, "A stochastic SIRS epidemic model with nonlinear incidence rate," Applied Mathematics and Computation, vol. 305, pp. 221-240, 2017.

[28] R. Z. Has'minskii, Stochastic Stability of Differential Equations, Springer, Berlin, Germany, 2011.

[29] L. R. Bellet, "Ergodic properties of markov process," in Open Quantum System II, Springer, Berlin, Germany, 2012.
[30] X. Mao, G. Marion, and E. Renshaw, "Environmental Brownian noise suppresses explosions in population dynamics," Stochastic Processes and Their Applications, vol. 97, no. 1, pp. 95-110, 2002.

[31] X. Mao, Stochastic Differential Equations and Their Applications, Horwood, Chichester, UK, 1997.

[32] Y. Zhao and D. Jiang, "The threshold of a stochastic SIS epidemic model with vaccination," Applied Mathematics and Computation, vol. 243, pp. 718-727, 2014.

[33] D. J. Higham, "An algorithmic introduction to numerical simulation of stochastic differential equations," SIAM Review, vol. 43, no. 3, pp. 525-546, 2001.

[34] E. Kussell and S. Leibler, "Phenotypic diversity, population growth, and information in fluctuating environments," Science, vol. 309, no. 5743, pp. 2075-2078, 2005.

[35] M. Liu, X. He, and J. Yu, "Dynamics of a stochastic regimeswitching predator-prey model with harvesting and distributed delays," Nonlinear Analysis: Hybrid Systems, vol. 28, pp. 87-104, 2018.

[36] M. Liu, J. Yu, and P. S. Mandal, "Dynamics of a stochastic delay competitive model with harvesting and Markovian switching," Applied Mathematics and Computation, vol. 337, pp. 335-349, 2018.

[37] M. Liu and Y. Zhu, "Stability of a budworm growth model with random perturbations," Applied Mathematics Letters, vol. 79, pp. 13-19, 2018. 


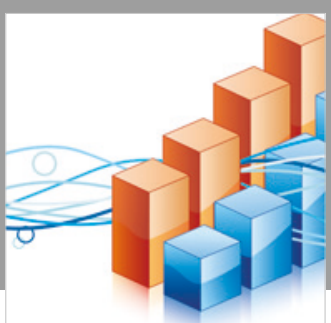

Advances in

Operations Research

\section{-n-m}
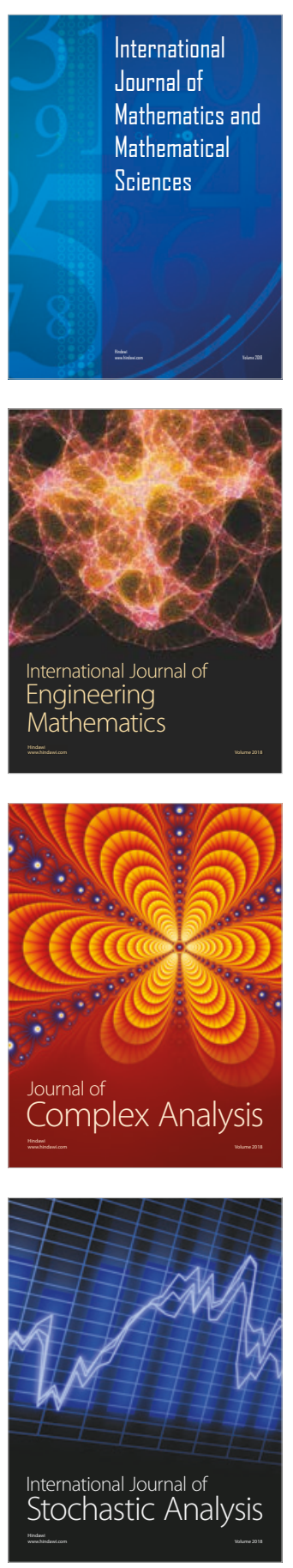
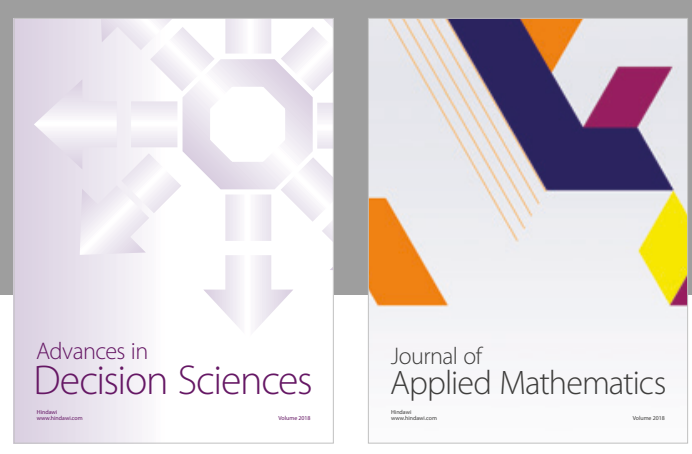

Journal of

Applied Mathematics
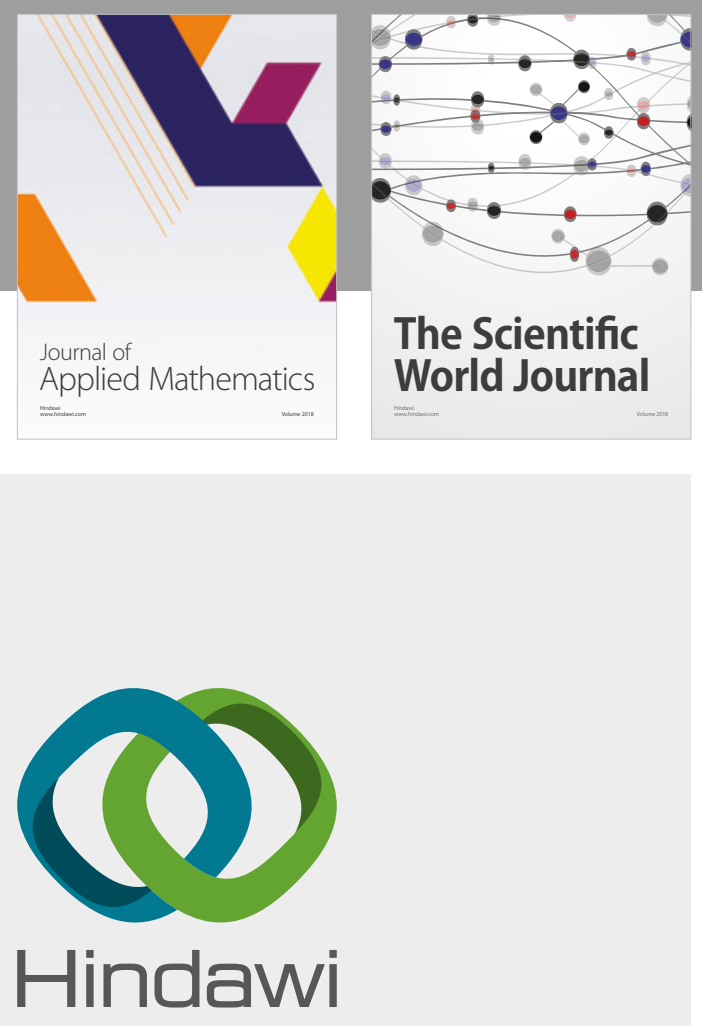

Submit your manuscripts at

www.hindawi.com

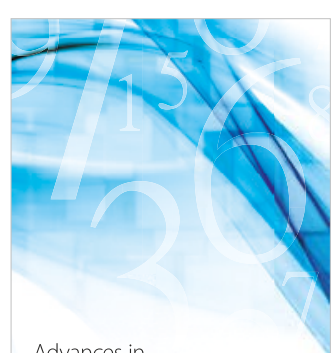

Advances in
Numerical Analysis
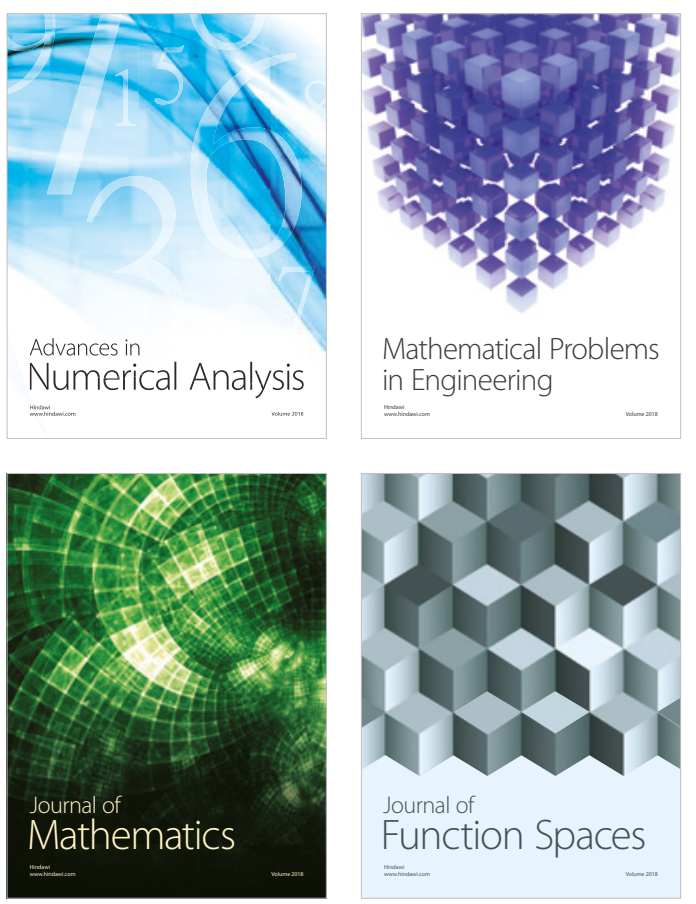

Mathematical Problems in Engineering

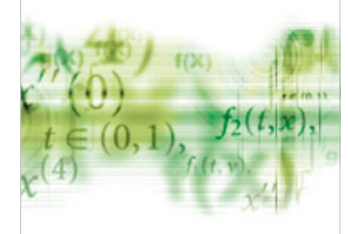

International Journal of

Differential Equations

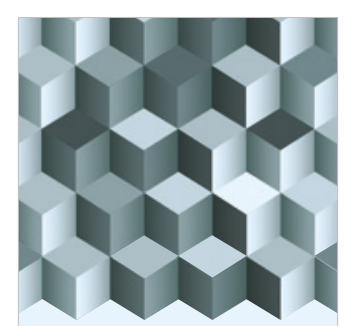

Journal of

Function Spaces
The Scientific

World Journal

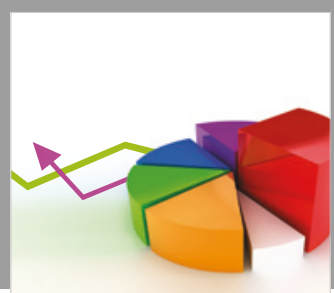

Journal of

Probability and Statistics
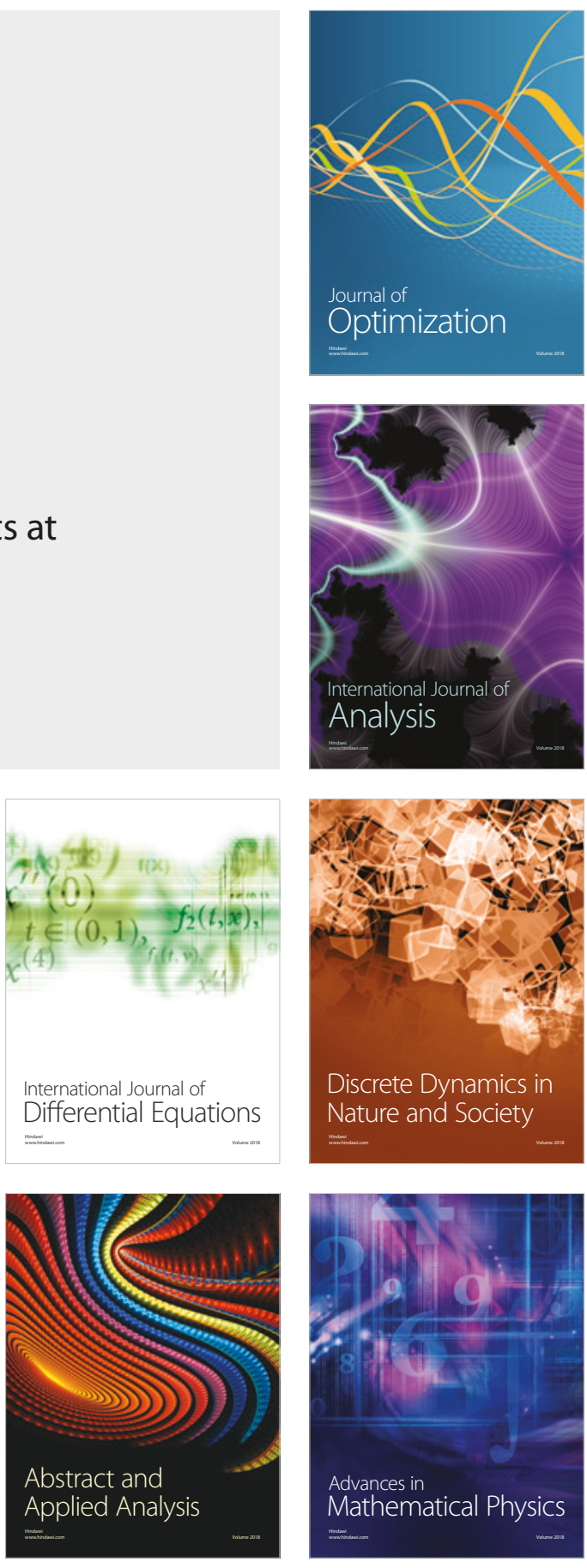